\title{
"Y" Variant of Double Cystic Duct: Incidental Finding During laparoscopic Cholecystectomy
}

\author{
Sunil Sadruddin Samnani • Aun Ali
}

Received: 3 October 2013 / Accepted: 27 March 2014 / Published online: 11 April 2014

(C) Association of Surgeons of India 2014

\section{Dear Editor,}

This correspondence is to discuss an incidental finding identified while conducting laparoscopic cholecystectomy. A 34year-old female visited the outpatient department of a tertiary hospital of Karachi, Pakistan. She had a history of on and off pain at the epigastric region for past 8 months. Physical examination revealed no significant findings. Her laboratory findings were within normal limits. Abdominal ultrasonography showed multiple stones in the gallbladder with the largest one measuring $1.5 \mathrm{~cm}$ in size, normal wall of gall bladder. Common bile duct (CBD) and portal vein size are within normal limits. No anatomic variation was highlighted at ultrasound.

A gallstone with occasional biliary colic was diagnosed, and the patient was planned for elective laparoscopic cholecystectomy. Perioperatively, dual cystic ducts were identified, clipped, and divided. It was found that the divided cystic duct contained two different channels. When the gallbladder was excised and examined, it was confirmed that the patient had two cystic ducts which were exiting from the gallbladder. Intraoperatively, the liver bed and clipped cystic duct stump was reexamined for any leakage. No leakage was found so drain was not placed. Postoperatively, the patient recovered well and discharged on the next postoperative day. The patient was followed up to 3 months and no complications were noticed.

Presence of variations particularly findings of double cystic duct during laparoscopic cholecystectomy is of great challenge for laparoscopic surgeons. In the past, only nine cases were found on this rare variant i.e., double cystic duct $[1,2]$. This anomaly is classified

S. S. Samnani $(\square) \cdot$ A. Ali

Department of Surgery, Jinnah Medical College Hospital,

SR-6, Sec-7-A, Korangi, Ind Area, Karachi, Pakistan

e-mail: dr.sunilsamnani@gmail.com by Flannery and Caster into three different types. The "H type" variant is common and mostly has published findings in previous case reports. The letter $\mathrm{H}$ represents the accessory cystic duct when it divides into three branches while it enters in the right, left, and common hepatic duct. In previous case reports published, accessory cystic duct opens into the right hepatic duct $[1,2]$. Another variant is the "Y type" in which two cystic duct opens into a common channel that joins the common hepatic duct. Our patient had the Y type variant with one lumen patent and the other obliterated. The last type is the trabecular type in which the cystic duct opens into substance of liver [3].

Intraoperative cholangiography is indicated to differentiate bile duct injury that might occur either because of transection of bile duct or because of the presence of anatomical variations. Careful examination is required to avoid conversions from laparoscopic to open cholecystectomy [1, 2]. In our case, we carefully examined the excised cystic duct as well as the liver bed and cystic duct stump. No leakage was identified. Hence, surgeons practicing in the laparoscopic era should be aware of anatomic variants and options available to avoid major complications.

\section{References}

1. Bernard P, Letessier E, Denimal F, Le Néel JC (2001) Accessory cystic duct discovered by intraoperative cholangiography during cholecystectomy. Ann Chir 126:1020-1022

2. Tsutsumi S, Hosouchi Y, Shimura T, Asao T, Kojima T, Takenoshita S, Kuwano H (2000) Double cystic duct detected by endoscopic retrograde cholangiopancreatography and confirmed by intraoperative cholangiography in laparoscopic cholecystectomy. Hepatogastroenterology 47:12661268

3. Bensen EA, Page RE (1976) A practical reappraisal of the anatomy of the extrahepatic bile duct and arteries. Br J Surg 63:853-860 